\title{
Quality of Service Routing in Mobile Ad hoc Networks Using Node Mobility and Energy Depletion Parameters
}

\author{
S.Prabhakara Rao ${ }^{1}$, Dr.E.Nagabhooshanam ${ }^{2}$, and S.Ramesh Babu ${ }^{3}$ \\ ${ }^{1}$ Assoc. Professor, Nigama Engineering College, Karimnagar, Andhra Pradesh \\ prabhakar367@gmail.com \\ ${ }^{2}$ Professor \& HOD, Sridevi Womens Engineering College, Gandipet, Hyderabad. \\ enb1234@rediffmail.com \\ ${ }^{3}$ Assoc. Professor, Nigama Engineering College, karimnagar \\ ramesh_sag@rediffmail.com
}

\begin{abstract}
:
Exceptionally dynamic networks are Mobile Ad hoc Networks. Quality of Service (QoS) routing in such Networks are frequently limited by the network split due to either energy depletion or node mobility of the mobile nodes. In addition, to fulfill specific quality parameters, existence of multiple node-disjoint paths becomes essential. Such paths assist in the optimal traffic distribution and consistency in case of path breakages. Thus, to accommodate such problem, we present a node-disjoint multipath protocol. The metric system of measurement used to select the paths takes into account the stability of the nodes and the equivalent links.
\end{abstract}

\section{KEYWORDS:}

QoS Routing, Mobile Ad hoc Networks, Energy-Aware Routing, Multipath Routing

\section{INTRODUCTION:}

Set of mobile or semi mobile nodes with no available pre-established communications is a MANET Forming a short-term network. Each mobile node in the network acts as a computer switching program that transfers incoming messages to outgoing links via the most efficient route possible, e.g. over the Internet i.e., a router. This kind of networks are characterize by the relationships between parts linked together in a system such as a computer network topologies, continuation of bandwidth constrain and variable capacity links, energy constrain operations and are highly intensity to security threats. Due to all these characteristics routing is a major issue in ad hoc networks. The routing protocols for ad hoc networks have been classified as: (a) Proactive or table driven for example Destination Sequenced Distance Vector (DSDV) and Optimized Link State Routing (OLSR), (b) Reactive/On-demand, e.g. Dynamic Source Routing Protocol, Ad hoc On-Demand Distance Vector routing protocol, Temporally Ordered Routing Algorithm. In table driven or proactive routing, each node has one or more tables that include the latest information of the routes to any node in the network. Each row has the subsequently hop for reaching to a node or subnet and the cost of this route. Different table-driven protocols vary in the way the information about alter in topology is spread through all nodes in the network. The two kinds of table keep informed in proactive protocols are the periodic update and the triggered update. Proactive routing has a movement to waste bandwidth and powers in the network due to the need to 
broadcast the routing tables are updates. In addition, as the numbers of nodes in the MANET expand, the size of the table will expand; this can become a problem in and of itself. In Reactive or On-demand, they do not retain or constantly update their route tables with the latest route topology. As an alternative, when a source node wants to transmit a message, it floods an uncertainty into the network to discover the route to the target. The un-covered path is maintained until the destination node becomes unreachable or until the route is not preferred anymore. The protocols in this class alter in handling supply routes and in the way route detection and route respond are holds. Reactive protocols are generally considered capable when the route discovery is employed rather rarely in comparison to the data transfer. Even though the network topology changes actively, the network traffic caused by the route discovery step is small compared to the total communication bandwidth. Hybrid Ad hoc Routing Protocol and Zone Routing Protocol are the proactive and reactive protocols effort well for networks with a small number of numbers of nodes. At the same time as the number of nodes increase, hybrid reactive or proactive protocols are used to achieve superior performance. Hybrid protocols effort to absorb the advantages of purely proactive and reactive protocols. The main idea is to use a reactive routing method at the worldwide network phase while make use of a proactive routing process in a node's local section. Quality of Service support routing is defined as a Routing mechanism under which paths for stream are compact based on some knowledge of provide skill in the network in addition to the Quality of Service requirement of flows. The main objectives of QoS based routing are :Dynamic determination of possible paths for accommodating the QoS of the given flow under policy control such as path cost, provider selection etc, optimal utilization of resources for improving total network throughput and smart performance degradation during overload conditions giving better throughput. QoS routing approach are classified as source routing distributed routing and hierarchical routing. QoS based routing becomes difficult in MANETs, as nodes should keep well developed information about link position. Moreover, due to the dynamic nature of MANETs, maintain the accurate link state information is very difficult. Finally, the reserved resource may not be guaranteed because of the mobility caused path breakage or power exhaustion of the mobile hosts. QoS routing should quickly find a possible new route to recover the service. The main aim in this paper is to plan a routing technique, which believes all three above problems seeing that one. To describe a metric that challenge to preserve equilibrium between mobility and energy boundary in Mobile Ad-hoc Networks.

\section{RELATED WORKS:}

During most recent period maximum research has been done in quality of service based, multipath and node disjoint routing. Recently, the forthcoming anxiety is the energy issues in mobile ad hoc networks. The modern learning expansively attentive on the multipath discovering addition of the On-demand routing protocols in order to develop single-path problems like AODV and DSR, such as large route detection latency, frequent route detection effort and probable improvement of data transport throughput. The multipath extension of AODV is AODVM. These provide loop free paths and link-disjoint in AODV. CM-AODV (Cross-layered multipath AODV), prefer multiple routes on demand position on the SINR (signal-to-interference plus noise ratio) , measured at the physical layer. The Multipath Source Routing (MSR) protocol is a multipath extension to DSR uses weighted round robin packet allotment to improve the delay and throughput. One more extension of DSR is SMR (Split Multipath Routing), which selects hop count partial and maximally disjoints many paths. Node-disjoint multiple paths provided by Node-Disjoint Multipath Routing (NDMR). Energy Aware Source Routing (EASR), Grid-based 
Energy Aware Node-Disjoint Multipath Routing Algorithm (GEANDMRA) and Energy Aware Node Disjoint multipath Routing (ENDMR) are further energy aware multipath protocols which give disjoint paths. The Lifetime-Aware Multipath Optimized Routing (LAMOR) is based on the lifetime of a node which is related to its outstanding energy and current traffic conditions. The combination of Minimum Transmission Power Routing (MTPR) and Power-aware Source Routing (PSR) provide Cost- effective Lifetime Prediction based Routing (CLPR), combines cost efficient and lifetime predictions based routing. Recently, the upcoming concern is the energy problem in mobile ad hoc networks (MANETs). The current studies broadly focused on the multipath determine the extension of the on- demand routing protocols in order to minimize the single-path problems like AODV and DSR, such as high route detection latency, frequent route discovery effort and possible improvement of data transfer throughput. The Multipath Source Routing (MSR) protocol is a multipath extension to DSR uses weighted round robin packet distribution to improve the delay and throughput. Cost- effective Lifetime Prediction based Routing (CLPR), combines cost efficient and lifetime predictions based routing. Minimum Transmission Power Routing (MTPR), Power-aware Source Routing (PSR).

\subsection{Dynamic Source Routing Protocol (DSR):}

Dynamic Source Routing is a reactive unicast (a transmission from a single computing terminal to one other terminal) routing protocol that make use of source routing algorithm. In this algorithm each data packet encloses complete routing information to reach its destination. Route cache is maintains by each node in DSR to maintain route information that it has learnt. The route discovery phase and the route maintenance phase are two major phases in DSR; it firstly checks its route cache when a source node wants to send a packet. The source node contains the routing information within the data packet before allocating it, if the required route is available. If not, the source node commences a route discovery process by broadcasting route request packets. The addresses of both the source and the destination and also a unique number to identify the request contain by a route request packet. In receipt of a route request packet, a node verifies its route cache. Node connects its own address to the route record field of the route request packet, if the it doesn't have routing information for the requested destination. After that, the specified packet is forwarded to its next to another or very nearby destination. A node evolution route request packets that both it has not seen before and its address is not existing in the route record field to bound the communication overhead of route request packets. A route respond packet is generated, for the route request packet to reach the destination or an in-between node has routing information to the destination. Route reply consists of addresses of nodes that have been passing through by the route request packet, when the route reply packet is generated by the destination. If not, the route reply packets contain the concentrate on of nodes the route request packet has pass through concatenated with the route in the midway node's route cache.

\section{PROBLEM ISSUE:}

In Mobile Ad hoc Networks Nodes are battery driven. Thus, they suffer from partial energy level problems. In the network the nodes are exciting, when a node moves out of the radio range of the other node, the link between them is not working. To achieve the route stability in MANETs, uniformly link stability and node stability is essential. As a result, in such an environment there are two main source of a link breakage: 1) Node moving out of the radio range of its adjacent 
node, 2) Node disappearing of energy collapse. Lifetime of a node utilize as a general metric, on the other hand it does not considers the mobility and energy concern delay are dangerous to network - lifetime assessment. The over mentioned techniques consider either of the two issues. Techniques in calculate only multiple paths. Both stability issues are neglected in these. The work in make sure route quality in terms of SINR, which gives reliable links, but overall networks constancy is not considered. Though uses lifetime of a node as a generalized metric, it does not considers the mobility and energy issues delay are critical to network - lifetime estimation. The lifetime of the node and mobility factor is not consider, only energy metric(relating to or using the metric system of measurement) to route the traffic, but the protocol in observe as the energy matter in terms of the energy expenses in data transmission. By using link disjoint or nodedisjoint, we can send a packet from a source to destination. The advantage of Node disjoints protocols that they avoid the rapid energy drainage of a node which is the portion of multiple link disjoint paths. Considering both link stability and node stability has been proposed, to find multiple node-disjoint paths. The challenge is to establish multiple node disjoint routes which consider both the node stability and link stability on their technique. By using Link Expiration Time (LET) we can measure link stability and Energy Drain Rate (EDR) we can measure node stability together respectively. Link Expiration Time (LET) and Energy Drain Rate (EDR) are two metrics can be used to generate a composite metric which maintain track of the stability level of the complete path.

\subsection{Mobility Factor:}

Link Expiration Time (LET) of mobility factor was proposed in by using the motion parameters (velocity (v), direction (d)) of the nodes. If $r$ is the Transmission distance between the two nodes, the position coordinates are i and j, (xi, yi) and (xj, yj) and (vi,_i) and (vj, _j) be the (velocity, direction) of motion of nodes. LET is defined as:

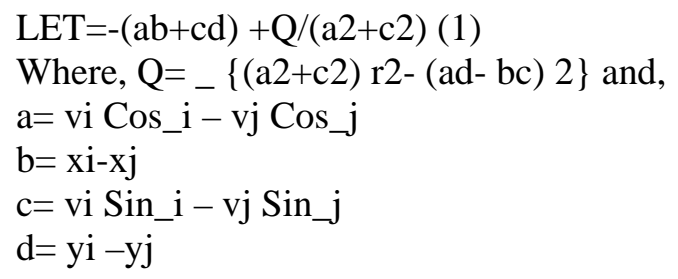

Through GPS, the movement parameters are replace among nodes at regular time intervals, the link will remain forever, as, LET will be if the two nodes have zero relative velocity, i,e, vi =vj and $\_j=\_$.

\subsection{Energy factor:}

Most of the energy based routing algorithms; send large volume of data on the route with maximum energy levels, As a result, nodes with much higher. The shift parameters are exchanged among nodes at regular time intervals through GPS. The above parameter propose that if the two nodes have zero relative velocity, $i, e, v i=v j$ and $\_j=\_j$. the link will remain forever, as, LET will be. The majority of the energy support routing algorithms; send large volume of data on the route with maximum energy levels, therefore, nodes with much higher current energy levels will be tired of their battery power very in the early hours. Due to pay concentration in of the next nodes, 
the mobile node also drop some of it energy. Even if no data is being sent through it, a node is losing its power over a period of time. Viewing of all these factors a metric (relating to or using the metric system of measurement) called Drain Rate (DR) was proposed. The Drain Rate of a node is defined as the rate of excess of energy of a node. Every node calculates its total energy usage every T-sec and estimates the Drain Rate (DR), Accurate Drain Rate is calculating by exponentially averaging the values of DR (Drain Rate) old and DR (Drain Rate) new as follows:

DR i=_DR old+ (1-_)DR new (2)

Where, $0<_{-}<$l, can be selected so as to give top priority to updated information.

Thus, higher the Drain Rate, faster the node exhausted of its energy.

\section{PROPOSED WORK:}

The central aim of the proposed work is to find the several node disjoint routes from source to a given destination. All the links of the routes are extremely protected in such way select the routes. This will amplify the lifetime of the route. In addition, it maintain path of the route bandwidth which can be improvement used by the source to select the optimal routes. From the factors Link Expiration Time (LET) [19] and Drain Rate (DR), it is conditional that the Link Stability depends directly on Mobility factor and inversely on the energy factor. Therefore, Link Stability Degree (LSD) = Mobility factor is divided by Energy factor. It defines the level of the stability of the link. Higher the stability of the link when Higher the value of LSD, and also larger the duration of its existence, if Higher the value of LSD. Therefore, a route contain all the links with LSD> LSD thru is the feasible route. We choose the Dynamic Source Routing (DSR) protocol as a candidate protocol. To allow the innovation of link stable node disjoint paths, modifications are made in the Route Request (RREQ) and Route Reply (RREP) packets. The proposed Scheme has three phases:

1. Route Discovery

2. Route Selection

3. Route preservation.

The different phases are illustrated as follows:

\subsection{Route Discovery:}

The source node when needs to send packet to some destination node, starts the route discovery procedure by sending the Route Request packet to all its neighbors .In this strategy , the source is not allowed to maintain route cache for a long time, as network conditions change very frequently in terms of position and energy levels of the nodes. Thus, when a nodes needs route to the destination, it initiates a Route Request packet, which is broadcasted to all the neighbors which satisfy the broadcasting condition. The Route Request(RREQ) packet of the DSR [5] is extended as RREQ of the NDMLNR adding two extra fields, LSD and Bandwidth, The combination of type field, source address field, destination field, unique identification number field, hop field, LSD, Bandwidth (cumulative bandwidth), Time -to-Live field and path field and also Type (T) field is nothing but RREQ. The field indicates the type of packet is Type (T) field. The field which carries the source address of node is SA (Source Address) field. Unique identification 
number generated by source to identify the packet is ID field. The field which carries the destination address of node is called DA (Destination Address) field. The field is used to limit the life time of packet is nothing but Time to Live (TTL) field, initially, by default it contains zero. LSD field: When packet passes through a node, its LSD value with the node from which it has received this packet is updated in the LSD field. Initially, by default this field contains zero value. Bandwidth field: carries the cumulative bandwidth of the links through which it passes; initially, by default this field contains zero value. The Hop field holds the hop count; the value of hop count is incremented by one for each node during packet passes, by default this field contains zero value. Path field carries the path accumulation, when packet passes through a node; its address is attached at end of this field. The route request packet RREQ packet contain SA, DA, Type(T), ID, TTL, Hop, LSD Path. The Route Reply packet (RREP) of the DSR is extended as RREP of the NDMLNR adding Bandwidth field. It is sent by the destination node after selecting the node disjoint paths among the various RREQ packets reaching it.

\subsection{Route Discovery at Intermediate node:}

Once an intermediate node receives a RREQ packet, it verifies whether its own address is previously listed in the path list of received RREQ packet or not by using DSR protocol. If its address is not found, it attach its address to the route record of received RREQ and it is broadcasted to all its neighbors. Otherwise, the received RREQ packet will be dropped. Since an intermediate node receives a RREQ packet, the NDMLNR, performs the tasks: 1 . it verify whether its own address is already listed in the route record of received RREQ packet. If its address is not found, it appends its address to the path list. 2. When an intermediate node receives a RREQ for the first time, it introduces a Wait Period, W. for the subsequent packets if any, with same identification number, traveling through different paths. It updates the value of LSD corresponding to the link on which it received the RREQ packet in the LSD field. It then checks its neighbors for QoS parameters, bandwidth here. Only those neighbors having LSD> LSD thr and Link Bandwidth $>=\mathrm{B}$ are considered for broadcasting. Once the neighbors with required LSD are selected, node forwards packet. Later if an intermediate node receives duplicate RREQ packets with same (Source address and ID), as received from other paths, those duplicate RREQ packets will be dropped. Every node maintains a Neighbor Information Table (NIT), to keep track of multiple RREQs. With following entries Source Address, Destination Address, Hops, LSD, ID and bandwidth. Neighbor Information Table (NIT) SA DA ID Hops LSD Bandwidth as a RREQ reaches a node it enters its information in the NIT. It makes all the entries for the requests till Wait Period. It agrees to the request with the highest value in LSD field at the end of the Wait Period. It adds the value of the link bandwidth to the Bandwidth field of the RREQ packet. If two RREQs have same LSD values, the one with lesser value of hop count is selected. In case, hops are also same, one with higher bandwidth is selected. In the worst case, RREQ is selected on First-come-first -serve basis. This prevents loops and unnecessary Flooding of RREQ packets. None of the intermediate nodes is allowed to send RREP if it has the current route to the destination. As doing this may lead to those paths which do not fulfill current QoS requirements.

\subsection{Destination node:}

In the NDMLNR, when the destination receives multiple RREQs it selects the paths with disjoint nodes. It then generates several replies and unicasts them to the source. Before that it appends its address and adds total bandwidth to each route request. Now each route reply that reaches the 
source contains a node-disjoint path from source to destination. Hence, source knows all the paths to the destination and their respective bandwidths. In case of two paths with one or more nodes common, the path with higher bandwidth is selected.

\subsection{Route Selection:}

When the source node receives the RREPs from the multiple paths, it sorts the paths in the order of the increasing bandwidth. Depending on the bandwidth the source decides to use the single path, or all of the paths. In case of the multiple paths with same bandwidths, path with minimum number of hops is selected. If the paths conflict on the number of hops, the source node selects the path on First-come-First-Serve basis.

\subsection{Route maintenance:}

In case, LSD of a node falls below LSD thru, it informs its predecessor node of the node failure by sending the NODEOFF message. Once a node receives such a message, it sends the ROUTEDISABLE message to the source node. Source can then reroute the packets to the backup routes. If no backup route exists, the source then starts the route discovery procedure again. We explain this technique with a suitable example in next section.

\section{CONCLUSION:}

The above mentioned technique considers the stability of the network from all aspects. The lifetime of the network can be reduced primarily by two causes. First, the node moving out of the radio range can lead to link breakage. Second, the node can be drained of its energy leading to network partitioning. The metric used in the proposed technique measures the stability of the network based on these two factors. The routing decisions at each node leads to the multiple paths, which are node disjoint. Doing this we attempt to prevent over usage of a single path nodes of which may drain out soon. Consequently, this technique is .likely to present highly constant, consistent, healthy node disjoint paths. As the paths are node disjoint, energy drain rate of the nodes is expected to be less and hence longer lifetime. Also the paths are selected on the bandwidth constraints; they are the ones with higher capacity. Thus in this technique, as the routes are selected completely satisfying stability and capacity constraints, it fully complies with Quality of Service objectives.

\section{REFERENCES:}

[1] D. D. Perkins, H. D. Hughes \& C. B. Owen, (2002) "Factors Affecting the Performance of Ad Hoc Networks,” Proceedings of the IEEE International Conference on Communications (ICC),2002, pp.2048-2052.

[2] Imrich Chlamtac, Marco Conti, Jennifer J.-N. Liu, (2003)" Mobile Ad hoc networking imperatives and challenges" Ad Hoc Networks, Vol 1, pp.13-64.

[3] C.E. Perkins \& P. Bhagwat (1994)" Highly Dynamic Destination-Sequenced Distance Vector Routing (DSDV) for Mobile Computers", ACM SIGCOMM Conference on Communications Architectures, Protocols and Applications, Vol. 24, pp. 234-244.

[4] M. Abolhasan, T.A. Wysocki, \& E. Dutkiewicz, (2004) "A Review of Routing Protocols for Mobile Ad hoc Networks", Ad hoc Networks, Vol. 2, pp. 1-2 International Journal of Wireless \& Mobile Networks (IJWMN), Vol 1, No 2, November 2009146 
International Journal of Network Security \& Its Applications (IJNSA), Vol.5, No.3, May 2013

[5] David B. Johnson, David A. Maltz, \& Josh Broch, "DSR: The Dynamic Source Routing Protocol for Multi-Hop Wireless Ad Hoc Networks", (2001), Ad Hoc Networking, Addison- Wesley , pp. 139172.

[6] C. E. Perkins \& E. M. Royer,(2003) "Ad hoc On-Demand Distance Vector Routing (AODV) ", IETF RFC 3561

[7] Z. Hass \& R. Pearlmann, "Zone routing Protocol"(1999), IETF Internet Draft

[8] RFC2386.

[9] Shigang Chen \& Klara Nahrstedt, (1998) ".An Overview of Quality-of-Service Routing for thenext Generation High -Speed Networks: Problems and Solutions", IEEE Network Magazine, vol12, pp. 64 $-79$.

[10] M. K. Marina \& S. R. Das, (2001) "On-Demand MultiPath Distance Vector Routing in Ad hoc Networks",Proceedings of the Ninth International Conference on Network Protocols (ICNP\},IEEE Computer Society Press, pp. 14-23.

[11] Jiwon Park, Sangman Moht \& Ilyong Chung (2008),” Multipath AODV Routing Protocol in Mobile Ad Hoc Networks with SINR-Based Route Selection”, International Symposium on Wireless Communication Systems, IEEE ,pp:682-688.

[12] Lei Wang, Lianfang Zhang, Yantai Shu \& Miao Dong (2000) ,” Multipath source routing in wireless ad hoc networks”, Proceedings of Canadian Conference on Electrical and Computer Engineering, Vol 1, pp. 479-483.

[13] S. J. Lee and M. Gerla (2001) "Split Multipath Routing with Maximally Disjoint Paths in Ad hoc Networks”,Proceedings of the IEEE International Conference on Communications(ICC),Vol 10, pp. 3201-3205.

[14] Dr. Shuchita Upadhayaya and Charu Gandhi “Node Disjoint Multipath Routing Considering Link And Node Stability Protocol: A Characteristic Evaluation” IJCSI International Journal of Computer Science Issues, Vol. 7, Issue 1, No. 2, January 2010, pp 18-25.

[15] Dr. Shuchita Upadhayaya And Charu Gandhi "Quality Of Service Routing Inmobile Ad Hoc Networks Using Location And Energy Parameters” International Journal Of Wireless \& Mobile Networks (IJWMN), Vol 1, No 2, November 2009,Pp 138 -147.

[16] Dr.Shuchita Upadhayaya, Charu Gandhi “Qos Routing Using Link And Node Stability In Mobile Ad Hoc Networks” Journal Of Theoretical And Applied Information Technology Pp 117-122. 